Communications in Physics, Vol. 20, No. 3 (2010), pp. 233-240

\title{
ENTANGLEMENT CRITERION FOR BIPARTITE QUANTUM STATES: APPLICATIONS
}

\author{
TRUONG MINH DUC AND NGUYEN THI XUAN HOAI \\ Department of Physics, Hue University of Pedagogy
}

\begin{abstract}
In this paper, we use a criterion provided by E. Shchukin and W. Vogel [Phys. Rev. Lett. 95, 230502 (2005)] for determining when a bipartite quantum state is entangled. We first show that the entanglement criterion is necessary and sufficient condition for the partial transposition of the bipartite quantum states. Furthermore, previously known entanglement criteria are proved to be special cases of this criterion. We then apply the entanglement criterion to determine entangled property of several non-classical two-mode states. Finally, we provide the conclusion about this criterion.
\end{abstract}

\section{INTRODUCTION}

For a recent review, it is believed that quantum entanglement plays an essential role in the rapidly developing field of quantum information processing [1]. An important matter is to check whether a quantum state is entangled. In order to solve this matter, Peres proposed an inseparable criterion based on negative partial transposition (NPT) of the composite density operator of the bipartite system [2]. However, with our best knowledge, characterizing NPT for such a system is still an completely unsolved problem.

The first set of inseparable criterions based on the NPT that was found is both necessary and sufficient conditions for the limited dimentional cases such as $2 \times 2,2 \times 3$, $3 \times 3$ and $2 \times 4$ systems [3,4]. After that, a sufficient condition for the NPT was proposed by Simon [5]. It is based on second-order moments of position and momentum operators and applied for the continuous variable systems. For the special case of Gaussian states, it becomes the necessary and sufficient condition. Another inseparable criteria based on second-order moments of observable quantities was derived without explicitly using the NPT $[6,7]$. These criteria are also complete conditions for two-mode Gaussian states and sufficient conditions for entanglement in any two-mode states. More recently, sufficient conditions for entanglement have been found for a wider class of states [8,9]. Especially, a criterion was provided by E. Shchukin and W. Vogel [9].

Our aim here is studying and exploring some of the implications of the ShchukinVogel entanglement criterion. We shall first show that this entanglement criterion is necessary and sufficient condition for the partial transposition of the bipartite quantum states. We shall then prove previously known entanglement criteria as special cases of this criterion. Finally, we shall use this criterion to detect entanglement of several non-classical two-mode states and provide the conclusion about it. 


\section{ENTANGLEMENT CRITERION FOR BIPARTITE QUANTUM STATES}

A Hermitian operator $\hat{A}$ is called non-negative if and only if for any operator $\hat{f}$ whose normally ordered form exists, the following inequality is satisfied

$$
\left\langle\hat{f}^{\dagger} \hat{f}\right\rangle_{\hat{A}}=\operatorname{Tr}\left(\hat{A} \hat{f}^{\dagger} \hat{f}\right) \geq 0 .
$$

Using for partially transposed density operator $\hat{\rho}^{P T}$, we give an inequality which is equivalent to the positive partial transposition (PPT)

$$
\left\langle f^{+} f\right\rangle^{P T}=\left\langle\hat{f}^{\dagger} \hat{f}\right\rangle_{\hat{\rho}^{P T}}=\operatorname{Tr}\left(\hat{\rho}^{P T} \hat{f}^{\dagger} \hat{f}\right) \geq 0 .
$$

For a form of operator $\hat{f}$

$$
\hat{f}=\sum_{n, m, k, l=0}^{+\infty} c_{n m k l} \hat{a}^{\dagger n} \hat{a}^{m} \hat{b}^{\dagger k} \hat{b}^{l},
$$

where $\hat{a}, \hat{b}$ are boson operators and $n, m, k, l$ are the presence of a number of annihilation or creation operator, the inequality (2) becomes

$$
\left\langle\hat{f}^{\dagger} \hat{f}\right\rangle^{P T}=\sum_{n, m, k, l, p, q, r, s=0}^{+\infty} c_{p q r s}^{*} c_{n m k l} M_{p q r s, n m k l} \geq 0
$$

where

$$
M_{p q r s, n m k l}=\left\langle\hat{a}^{\dagger q} \hat{a}^{p} \hat{a}^{\dagger n} \hat{a}^{m} \hat{b}^{\dagger s} \hat{b}^{r} \hat{b}^{\dagger k} \hat{b}^{l}\right\rangle^{P T} .
$$

According to the Sylvester criterion, the inequality (4) holds for all $c_{n m k l}$ if and only if all the subdeterminants of the form (4) are nonnegative. To be convenient, multi-indices $(n, m, k, l)$ and $(p, q, r, s)$ are respectively replaced by single-indices $u$ and $v$ with following convention

$$
u<v \Leftrightarrow \begin{cases}|u|<|v| & \text { or } \\ |u|=|v| & \text { and } u<^{\prime} v,\end{cases}
$$

where $|u|=n+m+k+l$ and $u<^{\prime} v$ means that the fisrt nonzero difference $r-k, s-$ $l, p-n, q-m$ is positive. According to this convention, the moments

$$
M_{p q r s, n m k l}^{0}=\left\langle\hat{a}^{\dagger q} \hat{a}^{p} \hat{a}^{\dagger n} \hat{a}^{m} \hat{b}^{\dagger s} \hat{b}^{r} \hat{b}^{\dagger k} \hat{b}^{l}\right\rangle
$$

are written with indices like matrix elements as follows

\begin{tabular}{|l|c|c|c|c|c|c|c|c|}
\hline $\mathrm{r}$ & $\mathrm{s}$ & $\mathrm{p}$ & $\mathrm{q}$ & $\mathrm{k}$ & $\mathrm{l}$ & $\mathrm{n}$ & $\mathrm{m}$ & $M_{i j}^{0}$ \\
\hline 0 & 0 & 0 & 0 & 0 & 0 & 0 & 0 & $M_{11}^{0}=1$ \\
\hline 0 & 0 & 0 & 0 & 0 & 0 & 0 & 1 & $M_{12}^{0}=\langle\hat{a}\rangle$ \\
\hline 0 & 0 & 0 & 0 & 0 & 0 & 1 & 0 & $M_{13}^{0}=\left\langle\hat{a}^{\dagger}\right\rangle$ \\
\hline$\ldots$ & $\ldots$ & $\ldots$ & $\ldots$ & $\ldots$ & $\ldots$ & $\ldots$ & $\ldots$ & $\ldots$ \\
\hline 0 & 0 & 0 & 0 & 0 & 0 & 0 & 2 & $M_{16}^{0}=\left\langle\hat{a}^{2}\right\rangle$ \\
\hline$\ldots$ & $\ldots$ & $\ldots$ & $\ldots$ & $\ldots$ & $\ldots$ & $\ldots$ & $\ldots$ & $\ldots$ \\
\hline 0 & 0 & 0 & 1 & 0 & 0 & 0 & 0 & $M_{21}^{0}=\left\langle\hat{a}^{\dagger}\right\rangle$ \\
\hline 0 & 0 & 0 & 1 & 0 & 0 & 0 & 1 & $M_{22}^{0}=\left\langle\hat{a}^{\dagger} \hat{a}\right\rangle$ \\
\hline$\ldots$ & $\ldots$ & $\ldots$ & $\ldots$ & $\ldots$ & $\ldots$ & $\ldots$ & $\ldots$ & $\ldots$ \\
\hline
\end{tabular}


Assuming that the partial transposition acts on the density matrix of the second partite then the partial transposition moments are represented in the form of initial moment as

$$
\left\langle\hat{a}^{\dagger q} \hat{a}^{p} \hat{a}^{\dagger n} \hat{a}^{m} \hat{b}^{\dagger s} \hat{b}^{r} \hat{b}^{\dagger k} \hat{b}^{l}\right\rangle^{P T}=\left\langle\hat{a}^{\dagger q} \hat{a}^{p} \hat{a}^{\dagger n} \hat{a}^{m} b^{\dagger l} \hat{b}^{k} \hat{b}^{\dagger r} \hat{b}^{s}\right\rangle .
$$

Now the necessary and sufficient condition for the PPT can be formulated

$$
D_{N}=\left|\begin{array}{cccc}
M_{11} & M_{12} & \ldots & M_{1 N} \\
M_{21} & M_{22} & \ldots & M_{2 N} \\
\ldots & \ldots & \ldots & \ldots \\
M_{N 1} & M_{N 2} & \ldots & M_{N N}
\end{array}\right| \geq 0, \quad \forall N
$$

where $M_{i j}$ is defined in Eq (5)and according to Eq (8) it is written

$$
M_{i j}=\left\langle\hat{a}^{\dagger q} \hat{a}^{p} \hat{a}^{\dagger n} \hat{a}^{m} \hat{b}^{\dagger l} \hat{b}^{k} \hat{b}^{\dagger r} \hat{b}^{s}\right\rangle=\left(M_{i j}^{0}\right)^{P T} .
$$

That is the entanglement criterion was proposed by Shchukin and Vogel [9]. It can be explicitly stated that: The partial transposition of a bipartite quantum state is nonnegative if and only if all the subdeterminants

$$
D_{N}=\left|\begin{array}{cccccc}
1 & \langle\hat{a}\rangle & \left\langle\hat{a}^{\dagger}\right\rangle & \left\langle\hat{b}^{\dagger}\right\rangle & \langle\hat{b}\rangle & \ldots \\
\left\langle\hat{a}^{\dagger}\right\rangle & \left\langle\hat{a}^{\dagger} \hat{a}\right\rangle & \left\langle\hat{a}^{\dagger 2}\right\rangle & \left\langle\hat{a}^{\dagger} \hat{b}^{\dagger}\right\rangle & \left\langle\hat{a}^{\dagger} \hat{b}\right\rangle & \ldots \\
\langle\hat{a}\rangle & \left\langle\hat{a}^{2}\right\rangle & \left\langle\hat{a} \hat{a}^{\dagger}\right\rangle & \left\langle\hat{a} \hat{b}^{\dagger}\right\rangle & \langle\hat{a} \hat{b}\rangle & \ldots \\
\langle\hat{b}\rangle & \langle\hat{a} \hat{b}\rangle & \left\langle\hat{a}^{\dagger} \hat{b}\right\rangle & \left\langle\hat{b}^{\dagger} \hat{b}\right\rangle & \left\langle\hat{b}^{2}\right\rangle & \ldots \\
\left\langle\hat{b}^{\dagger}\right\rangle & \left\langle\hat{a} \hat{b}^{\dagger}\right\rangle & \left\langle\hat{a}^{\dagger} \hat{b}^{\dagger}\right\rangle & \left\langle\hat{b}^{\dagger 2}\right\rangle & \left\langle\hat{b}^{\dagger}\right\rangle & \ldots \\
\ldots & \ldots & \ldots & \ldots & \ldots & \ldots
\end{array}\right|
$$

are nonnegative for all $N$. The other way around, if there exists a negative subdeterminant

$$
\exists N: \quad D_{N}<0,
$$

the partial transposition matrix defines negative. It means that the criterion (12) is a sufficient condition for determining entangled property of bipartite quantum systems.

As to be mentioned in Ref. [9], previously known entanglement criteria are proved to be special cases of this criterion. Now we shall prove this remark in detail. We start with the separable condition found by Simon [5]. For any separable quantum state, the quantity S

$$
S=\operatorname{det} A_{1} \operatorname{det} A_{2}+\left(\frac{1}{4}+\operatorname{det} C\right)^{2}-\operatorname{Tr}\left(A_{1} J C J A_{2} J C^{T} J\right)-\frac{1}{4}\left(\operatorname{det} A_{1}+\operatorname{det} A_{2}\right) \geq 0,
$$

where

$$
A_{i}=\left(\begin{array}{cc}
\left\langle\left(\Delta \hat{x}_{i}\right)^{2}\right\rangle & \left\langle\left\{\Delta \hat{x}_{i}, \Delta \hat{p}_{i}\right\}\right\rangle \\
\left\langle\left\{\Delta \hat{x}_{i}, \Delta \hat{p}_{i}\right\}\right\rangle & \left\langle\left(\Delta \hat{p}_{i}\right)^{2}\right\rangle
\end{array}\right), \quad C=\left(\begin{array}{cc}
\left\langle\Delta \hat{x}_{1} \Delta \hat{x}_{2}\right\rangle & \left\langle\Delta \hat{x}_{1} \Delta \hat{p}_{2}\right\rangle \\
\left\langle\Delta \hat{p}_{1} \Delta \hat{x}_{2}\right\rangle & \left\langle\Delta \hat{p}_{1} \Delta \hat{p}_{2}\right\rangle
\end{array}\right)
$$

with

and

$$
\left\langle\left\{\Delta \hat{q}_{i}, \Delta \hat{p}_{i}\right\}\right\rangle=\frac{1}{2}\left\langle\Delta \hat{q}_{i} \Delta \hat{p}_{i}+\Delta \hat{p}_{i} \Delta \hat{q}_{i}\right\rangle,
$$

$$
J=\left(\begin{array}{cc}
0 & 1 \\
-1 & 0
\end{array}\right)
$$


Expanding $\mathrm{S}$ in form the annihilation and creation operators, then comparing with a following subdeterminant of $D_{N}$

$$
D_{5}=\left|\begin{array}{ccccc}
1 & \langle\hat{a}\rangle & \left\langle\hat{a}^{\dagger}\right\rangle & \left\langle\hat{b}^{\dagger}\right\rangle & \langle\hat{b}\rangle \\
\left\langle\hat{a}^{\dagger}\right\rangle & \left\langle\hat{a}^{\dagger} \hat{a}\right\rangle & \left\langle\hat{a}^{\dagger 2}\right\rangle & \left\langle\hat{a}^{\dagger} \hat{b}^{\dagger}\right\rangle & \left\langle\hat{a}^{\dagger} \hat{b}\right\rangle \\
\langle\hat{a}\rangle & \left\langle\hat{a}^{2}\right\rangle & \left\langle\hat{a} \hat{a}^{\dagger}\right\rangle & \left\langle\hat{a} \hat{b}^{\dagger}\right\rangle & \langle\hat{a} \hat{b}\rangle \\
\langle\hat{b}\rangle & \langle\hat{a} \hat{b}\rangle & \left\langle\hat{a}^{\dagger} \hat{b}\right\rangle & \left\langle\hat{b}^{\dagger} \hat{b}\right\rangle & \left\langle\hat{b}^{2}\right\rangle \\
\left\langle\hat{b}^{\dagger}\right\rangle & \left\langle\hat{a} \hat{b}^{\dagger}\right\rangle & \left\langle\hat{a}^{\dagger} \hat{b}^{\dagger}\right\rangle & \left\langle\hat{b}^{\dagger 2}\right\rangle & \left\langle\hat{b} \hat{b}^{\dagger}\right\rangle
\end{array}\right|,
$$

we find that $S$ coincides with $D_{5}$. Hence, Simon criterion is only a special case of ShchukinVogel criterion.

Another separable condition was formulated in Ref. [6]. For any separable state, the inequality

$$
\left\langle(\Delta \hat{u})^{2}\right\rangle_{\rho}+\left\langle(\Delta \hat{v})^{2}\right\rangle_{\rho} \geq r^{2}+\frac{1}{r^{2}}
$$

holds true for all nonzero real parameter $r$, where $\hat{u}=|r| \hat{x}_{1}+\frac{1}{r} \hat{x}_{2}$ and $\hat{v}=|r| \hat{p}_{1}-\frac{1}{r} \hat{p}_{2}$. By representing operators $\hat{u}$ and $\hat{v}$ in form the creation and annihilation operators of the fisrt mode $\hat{a}^{\dagger}, \hat{a}$ and the second mode $\hat{b}^{\dagger}, \hat{b}$ we find

$$
\begin{aligned}
\left\langle(\Delta \hat{u})^{2}\right\rangle+ & \left\langle(\Delta \hat{v})^{2}\right\rangle \\
& =2 r^{2}\left\langle\Delta \hat{a}^{\dagger} \Delta \hat{a}\right\rangle+\frac{2}{r^{2}}\left\langle\Delta \hat{b}^{\dagger} \Delta \hat{b}\right\rangle+2 \frac{r}{|r|}\left[\langle\Delta \hat{a} \Delta \hat{b}\rangle+\left\langle\Delta \hat{a}^{\dagger} \Delta \hat{b}^{\dagger}\right\rangle\right]+r^{2}+\frac{1}{r^{2}} .
\end{aligned}
$$

Inserting (17) in (16) and noticing $\langle\Delta \hat{a} \Delta \hat{b}\rangle+\left\langle\Delta \hat{a}^{\dagger} \Delta \hat{b}^{\dagger}\right\rangle=2 R e\langle\Delta \hat{a} \Delta \hat{b}\rangle$ we have

$$
r^{4}\left\langle\Delta \hat{a}^{\dagger} \Delta \hat{a}\right\rangle+2 \frac{r^{3}}{|r|} \operatorname{Re}\langle\Delta \hat{a} \Delta \hat{b}\rangle+\left\langle\Delta \hat{b}^{\dagger} \Delta \hat{b}\right\rangle \geq 0 .
$$

Let the inequality (18) be true for all nonzero real parameter $r$ then

$$
\operatorname{Re}^{2}\langle\Delta \hat{a} \Delta \hat{b}\rangle \leq\left\langle\Delta \hat{a}^{\dagger} \Delta \hat{a}\right\rangle\left\langle\Delta \hat{b}^{\dagger} \Delta \hat{b}\right\rangle
$$

Considering a subdeterminant of $D_{N}$ as follows

$$
d=\left|\begin{array}{ccc}
1 & \langle\hat{a}\rangle & \left\langle\hat{b}^{\dagger}\right\rangle \\
\left\langle\hat{a}^{\dagger}\right\rangle & \left\langle\hat{a}^{\dagger} \hat{a}\right\rangle & \left\langle\hat{a}^{\dagger} \hat{b}^{\dagger}\right\rangle \\
\langle\hat{b}\rangle & \langle\hat{a} \hat{b}\rangle & \left\langle\hat{b}^{\dagger} \hat{b}\right\rangle
\end{array}\right| .
$$

We have

$$
\begin{aligned}
d= & \left\langle\hat{a}^{\dagger} \hat{a}\right\rangle\left\langle\hat{b}^{\dagger} \hat{b}\right\rangle+\left\langle\hat{a}^{\dagger}\right\rangle\left\langle\hat{b}^{\dagger}\right\rangle\langle\hat{a} \hat{b}\rangle+\left\langle\hat{a}^{\dagger} \hat{b}^{\dagger}\right\rangle\langle\hat{a}\rangle\langle\hat{b}\rangle \\
& -\left\langle\hat{a}^{\dagger} \hat{a}\right\rangle\left\langle\hat{b}^{\dagger}\right\rangle\langle\hat{b}\rangle-\left\langle\hat{a}^{\dagger}\right\rangle\langle\hat{a}\rangle\left\langle\hat{b}^{\dagger} \hat{b}\right\rangle-\left\langle\hat{a}^{\dagger} \hat{b}^{\dagger}\right\rangle\langle\hat{a} \hat{b}\rangle \\
= & \left\langle\Delta \hat{a}^{\dagger} \Delta \hat{a}\right\rangle\left\langle\Delta \hat{b}^{\dagger} \Delta \hat{b}\right\rangle-|\langle\Delta \hat{a} \Delta \hat{b}\rangle|^{2} .
\end{aligned}
$$

According to Eq. (21) we find $d \geq 0$ when

$$
\left\langle\Delta \hat{a}^{\dagger} \Delta \hat{a}\right\rangle\left\langle\Delta \hat{b}^{\dagger} \Delta \hat{b}\right\rangle \geq|\langle\Delta \hat{a} \Delta \hat{b}\rangle|^{2} .
$$

It becomes clear that the condition (19) is weaker than the condition (22). 
We now consider the condition derived recently in Ref. [8]: For any separable state, we have the inequality

$$
\left\langle\hat{a}^{\dagger m} \hat{a}^{m} \hat{b}^{\dagger n} \hat{b}^{n}\right\rangle \geq\left|\left\langle\hat{a}^{m} \hat{b}^{\dagger n}\right\rangle\right|^{2} .
$$

In Eq. (3), if we only retain two terms and identify other terms with zero, we have

$$
\hat{f}=C_{1} \hat{a}^{\dagger n} \hat{a}^{m} \hat{b}^{\dagger k} \hat{b}^{l}+C_{2} \hat{a}^{\dagger p} \hat{a}^{q} \hat{b}^{\dagger r} \hat{b}^{s} .
$$

Then Shchukin-Vogel criterion forms

$$
\begin{aligned}
\left\langle\hat{f}^{\dagger} \hat{f}\right\rangle^{P T}=\left\langle\hat{a}^{\dagger m} \hat{a}^{n} \hat{a}^{\dagger n} \hat{a}^{m} \hat{b}^{\dagger l} \hat{b}^{k} \hat{b}^{\dagger k} \hat{b}^{l}\right\rangle\left\langle\hat{a}^{\dagger q} \hat{a}^{p} \hat{a}^{\dagger p} \hat{a}^{q} \hat{b}^{\dagger s} \hat{b}^{r} \hat{b}^{\dagger r} \hat{b}^{s}\right\rangle & \\
& -\left\langle\hat{a}^{\dagger m} \hat{a}^{n} \hat{a}^{\dagger p} \hat{a}^{q} \hat{b}^{\dagger s} \hat{b}^{r} \hat{b}^{\dagger k} \hat{b}^{l}\right\rangle\left\langle\hat{a}^{\dagger q} \hat{a}^{p} \hat{a}^{\dagger n} \hat{a}^{m} \hat{b}^{\dagger l} \hat{b}^{k} \hat{b}^{\dagger r} \hat{b}^{s}\right\rangle \geq 0 .
\end{aligned}
$$

For $n=k=p=q=r=s=0$, the inequality (25) becomes

$$
\left\langle\hat{a}^{\dagger m} \hat{a}^{m} \hat{b}^{\dagger l} \hat{b}^{l}\right\rangle \geq\left|\left\langle\hat{a}^{m} \hat{b}^{\dagger l}\right\rangle\right|^{2} .
$$

By comparing (23) and (26) we find that for $\hat{f}$ is defined by Eq. (24), Shchukin-Vogel criterion coincides with Hillery-Zubairy criterion.

\section{APPLICATION}

In order to gain a better understanding of the applicability of this entanglement criterion, we shall study several examples. The fisrt example is that of a pair coherent state. As defined by Agarwal [10], a pair coherent state of a two mode radiation field is simultaneous eigenstate of the pair annihilation operator $\hat{a} \hat{b}$ and the difference in photon number operator $\hat{a}^{\dagger} \hat{a}-\hat{b}^{\dagger} \hat{b}$ :

$$
\begin{aligned}
\hat{a} \hat{b}|\xi, q\rangle & =\xi|\xi, q\rangle, \\
\left(\hat{a}^{\dagger} \hat{a}-\hat{b}^{\dagger} \hat{b}\right)|\xi, q\rangle & =q|\xi, q\rangle,
\end{aligned}
$$

where $\hat{a}$ and $\hat{b}$ are two annihilation operators associated with two modes. The pair coherent state is specified by two parameters, $\xi$ is a complex number and $q$, which is called the degeneracy parameter, takes on integer values and can be assumed to be positive without any loss of generality. This is the non-Gaussian entangled state and is written using the Fock basic as

$$
|\xi, q\rangle=N_{q} \sum_{n=0}^{\infty} \frac{\xi^{n}}{\sqrt{n !(n+q) !}}|n+q, n\rangle,
$$

where

$$
N_{q}=\left[\sum_{n=0}^{\infty} \frac{|\xi|^{2 n}}{n !(n+q) !}\right]^{-1 / 2}=\left[|\xi|^{-q} I_{q}(2|\xi|)\right]^{-1 / 2}
$$

is the normalization coefficient, $I_{q}(2|\xi|)$ being the modified Bessel function. 
According to Shchukin-Vogel criterion, we have a subdeterminant origined from operator $\hat{f}=c_{1}+c_{2} \hat{a}^{2}+c_{3} \hat{b}^{2}$ as following:

$$
D=\left|\begin{array}{ccc}
1 & \left\langle\hat{a}^{2}\right\rangle & \left\langle\hat{b}^{\dagger 2}\right\rangle \\
\left\langle\hat{a}^{\dagger 2}\right\rangle & \left\langle\hat{a}^{\dagger 2} \hat{a}^{2}\right\rangle & \left\langle\hat{a}^{\dagger 2} \hat{b}^{\dagger 2}\right\rangle \\
\left\langle\hat{b}^{2}\right\rangle & \left\langle\hat{a}^{2} \hat{b}^{2}\right\rangle & \left\langle\hat{b}^{\dagger 2} \hat{b}^{2}\right\rangle
\end{array}\right| .
$$

Applying this subdeterminant for the pair coherent states we accquire

$$
D=-x^{2}|\xi|^{4}\left[x^{2}\left(q^{2}-1\right)+2\right]-q|\xi|^{2}\left[x^{2} q^{2}-q+1-x^{2}\right] .
$$

For $q=0$, we find

$$
D_{0}=-\frac{N_{0}^{2}}{N_{1}^{2}}|\xi|^{4}\left(2-\frac{N_{0}^{2}}{N_{1}^{2}}\right) .
$$

It becomes clear that $D_{0}$ is negative for all values of $\xi$. In addition, for other parameters $q, D<0$ is still true. It means that the pair coherent states satisfy the inequality (12) and it is entangled. Whereas the criteria in Refs. [5-8] do not either provide sufficient conditions to determine the existence of entanglement or confirm that the pair coherent states are entangled [11].

As a second example, let us consider a symmetric superposition of two two-mode coherent states

$$
|\psi\rangle=\left(2+2 x^{2}\right)^{-1 / 2}(|\alpha, \beta\rangle+|\beta, \alpha\rangle),
$$

with $x=|\langle\alpha \mid \beta\rangle|$. When considering a subdeterminant derived from Schukin-Vogel by using operator $\hat{f}=c_{1}+c_{2} \hat{a}+c_{3} \hat{b}^{2}$ :

$$
D=\left|\begin{array}{ccc}
1 & \langle\hat{a}\rangle & \left\langle\hat{b}^{\dagger 2}\right\rangle \\
\left\langle\hat{a}^{\dagger}\right\rangle & \left\langle\hat{a}^{\dagger} \hat{a}\right\rangle & \left\langle\hat{a}^{\dagger} \hat{b}^{\dagger 2}\right\rangle \\
\left\langle\hat{b}^{2}\right\rangle & \left\langle\hat{a} \hat{b}^{2}\right\rangle & \left\langle\hat{b}^{\dagger 2} \hat{b}^{2}\right\rangle
\end{array}\right|,
$$

we obtain

$$
D=-\mathfrak{N}^{2} x^{2}\left[|\alpha|^{2}+|\beta|^{2}-\left(\alpha^{*} \beta+\alpha \beta^{*}\right)\right]^{2}|\alpha+\beta|^{2},
$$

with

$$
\mathfrak{N}=\left(2+2 x^{2}\right)^{-1} .
$$

According to (35) the symmetric superposition of two two-mode coherent states will be entangled for all $\alpha \neq \beta$. We will not gain this result with the criterion in Ref. $[5,7,8]$ again.

Finally, we consider a two mode photon-added coherent state

$$
|\psi\rangle_{a b}=\left(2+|\alpha+\beta|^{2}\right)^{-1 / 2}\left(a^{\dagger}+b^{\dagger}\right)|\alpha, \beta\rangle .
$$

We may apply a subdeterminant of $D_{N}$ to the one resulting from operator $\hat{f}=c_{1}+c_{2} \hat{a}+c_{3} \hat{b}$ :

$$
D=\left|\begin{array}{ccc}
1 & \langle\hat{a}\rangle & \left\langle\hat{b}^{\dagger}\right\rangle \\
\left\langle\hat{a}^{\dagger}\right\rangle & \left\langle\hat{a}^{\dagger} \hat{a}\right\rangle & \left\langle\hat{a}^{\dagger} \hat{b}^{\dagger}\right\rangle \\
\langle\hat{b}\rangle & \langle\hat{a} \hat{b}\rangle & \left\langle\hat{b}^{\dagger} \hat{b}\right\rangle
\end{array}\right| .
$$


For this state, it is convenient to use

$$
\left\langle\alpha\left|a^{k} a^{\dagger l}\right| \alpha\right\rangle= \begin{cases}k ! L_{k}^{l-k}\left(-|\alpha|^{2}\right) \alpha^{*^{(l-k)}} & \text { if } \quad l \geq k \\ l ! L_{l}^{k-l}\left(-|\alpha|^{2}\right) \alpha^{k-l} & \text { if } \quad k \geq l,\end{cases}
$$

where $L_{n}^{m}(x)$ is the Laguerre polynomial

$$
L_{n}^{m}(x)=\sum_{k=0}^{n} \frac{(m+n) !(-x)^{k}}{(m+k) !(n-k) ! k !} .
$$

By using (39) we get the expressions of the elements in the subdeterminant (38) and final result

where

$$
D=\mathfrak{N}_{\alpha \beta}^{3} \times\left(2-|\alpha+\beta|^{2}\right),
$$

$$
\mathfrak{N}_{\alpha \beta}=\left(2+|\alpha+\beta|^{2}\right)^{-1} .
$$

It can be realized that according to this criterion, the two-mode photon-added coherent state will be entangled for all $\alpha$ and $\beta$ satisfying $|\alpha+\beta|^{2}>2$. Comparing with result achieved in Ref. [12], it is clear the entanglement condition obtained in this paper is more easy to gain.

\section{CONCLUSION}

In conclusion, we have proved that several previously known entanglement criteria are special cases of the criterion in Ref. [9] in detail. Specially, we have used this criterion for finding entangled property of two mode nonclassical states. Our calculations yield the pair coherent state will be entangled for all $\xi$ for some value of $q$ parameters. This result would not gain if we used previously known entanglement criteria. Furthermore, for the symmetric superposition of two two-mode coherent states and the two mode photon added coherent state, the entanglement conditions calculated by this criterion also are stronger than one calculated by some known entanglement criteria. It means that the criterion studied in this paper is not only general but also stronger than previously known entan-

glement criteria. Hence, we have great expectations of the applicability of this criterion for determining when a bipartite quantum state is entangled. Finally, it is necessary to notice that we can use this criterion for higher mode bipartite quantum states.

\section{ACKNOWLEDGMENT}

The authors would like to thank Prof. Nguyen Ba An for his valuable comments. This work was supported by the NAFOSTED.

\section{REFERENCES}

[1] Samuel L.Braustein, Peter van Loock, Review of Modern Physics 77 (2005) 513.

[2] Asher Peres, Physical Review Letters 77 (1996) 1413.

[3] Michal Horodecki, Pawel Horodecki, Ryszard Horodecki, Phys. Lett. A223 (1996) 1.

[4] Pawel Horodecki, Physics Letter A, 232 (1997) 333.

[5] R. Simon, Physical Review Letters, 84 (2000) 2726. 
[6] L. M. Duan, G. Giedke, J. I. Cirac, P. Zoller, Physical Review Letters, 84 (2000) 2722.

[7] M. G. Raymer, A. C. Funk, B. C. Sander, H. Guise, Physics Review A, 67 (2003) 052104.

[8] Mark Hillery, M. Suhail Zubairy, Physical Review Letters, 96 (2006) 050503.

[9] E. Shchukin, W. Vogel, Physical Review Letters, 95 (2005) 230502.

[10] G. S. Agarwal, Journal of the Optical Society of America B, 5 (1988) 1940.

[11] T. M. Duc, J. Noh, K. Kim, Advances in Natural Sciences, 9 (2008) 107.

[12] Mark Hillery, M. Suhail Zubairy, Physical Review A, 74 (2006) 032333.

[13] A.Einstein, B.Podolsky and N.Rosen, Physics Review, 47 (1935) 777. 